EPJ Web of Conferences 47, 06002 (2013)

DOI: $10.1051 /$ epjconf/20134706002

(C) Owned by the authors, published by EDP Sciences, 2013

\title{
Searching for benchmark systems containing ultra-cool dwarfs and white dwarfs
}

\author{
J.I. Gomes ${ }^{\mathrm{a}}$ and D.J. Pinfield \\ Centre for Astrophysics Research, University of Hertfordshire
}

\begin{abstract}
We have used the 2MASS all-sky survey and WISE to look for ultracool dwarfs that are part of multiple systems containing main sequence stars. We cross-matched L dwarf candidates from the surveys with Hipparcos and Gliese stars, finding two new systems. We consider the binary fraction for L dwarfs and main sequence stars, and further assess possible unresolved multiplicity within the full companion sample. This analysis shows that some of the L dwarfs in this sample might actually be unresolved binaries themselves. We have also identified a sample of common proper motion systems in which a main sequence star has a white dwarf as wide companion. These systems can help explore key issues in star evolution theory, as the initial-final mass relationship of white dwarfs, or the chromospheric activity-age relationship for stars still in the main sequence. Spectroscopy for 50 white dwarf candidates, selected from the SuperCOSMOS Science Archive, was obtained. We have also observed 6 of the main sequence star companions, and have estimated their effective temperatures, rotational and microturbulent velocities and metallicities.
\end{abstract}

\section{ULTRA-COOL DWARFS AND MS STAR BINARIES}

The complexities of ultracool atmospheres are best confronted by observations of ultracool dwarfs (UCDs) with well known physical properties (luminosity, mass, effective temperature, surface gravity, and metallicity), so called "benchmark objects" [1]. Amongst the various types of benchmark objects, the ones that have a main sequence (MS) star as a wide companion are one of the most plentiful. Assuming that both components in the system are coeval, we can infer the properties of the secondary by studying the primary and see how their spectral energy distribution depends on physical properties. Also, since these are wide systems, we can consider that there has been no interaction (like mass exchange) between the components during their formation and evolution.

\subsection{Method}

We have used various large infrared sky surveys (2MASS, WIDE, UKIDSS and VISTA) to look for L dwarfs. This was done with the aid of several colour and magnitude cuts. We then cross-matched our initial list of $\mathrm{L}$ dwarf candidates with F, G and $\mathrm{K}$ stars from the Hipparcos and Gliese catalogs, taking into account factors as on-sky separations, consistency in derived distances and common proper motion. For the proper motion analysis we have used 2MASS and WISE epochs when available, since these surveys provide the largest baseline possible (approximately 10 years between the two epochs). The result is the discovery of two new benchmark systems (from WISE and 2MASS), presented in Gomes et al. (submitted).

\footnotetext{
ae-mail: j.gomes@herts.ac.uk
}

This is an Open Access article distributed under the terms of the Creative Commons Attribution License 2.0, which permits unrestricted use, distribution, and reproduction in any medium, provided the original work is properly cited. 


\section{EPJ Web of Conferences}

\subsection{Results}

The first binary, G255-34AB, consists of a new L2 dwarf with a K8 star as companion, at a distance of 36 pc. This L dwarf has been detected in WISE and W1 and W2 colours have been used to estimate its spectral type, later confirmed with spectroscopic observations taken with the DOLORES instrument, at the TNG telescope. The bolometric luminosity of this $\mathrm{L}$ dwarf is $1.67 \pm 0.17 \times 10^{-4} \mathrm{~L}_{\odot}$ and its temperature, considering a radius range of 0.8 to $1.2 R_{\text {Jup }}$, is $2080 \pm 260 \mathrm{~K}$.

The second discovery is the triple system GJ499ABC, with an L5 dwarf as a companion to a binary with an M4 and K5 stars. The spectral type of this L dwarf was already published in [2], who classified the source as L4 \pm 2 . Using spectra available in SDSS, and comparing it to template spectra (see [3]), we adopt a spectral type of L5.

We also present a list of the only 22 systems in which L dwarfs have MS stars as primaries, and estimate the binary fraction for a sample of L dwarfs within our completeness limit of $19 \mathrm{pc}$. We find that the binary fraction for L dwarfs that have MS companions is 6 per cent. On the other hand, the fraction of stars with $\mathrm{L}$ dwarfs as wide companions is only 0.33 per cent.

Another interesting result is highlighted with the help of a spectral type-magnitude diagram. In it, we show that some L dwarfs thought to be singular objects may in fact be unresolved binaries since they sit well above the expected position in the plot and are therefore brighter for the assumed spectral type. Further observations, using adaptive optics if possible, will help disentangle these results.

\section{WHITE DWARFS AND MS STAR BINARIES}

\subsection{Method}

Another set of interesting wide systems are the ones with MS stars and white dwarfs (WDs). As mentioned in section 1, we can consider that the components are independent, only sharing age and composition. Such systems have many applications in key areas of astronomy. WDs can be used as chronometers, giving system and stellar ages, a parameter not well constrained. Another valuable lane to be explored is the hypothesis that some WDs show polluted atmospheres not due to the interstellar medium accretion, but due to the accretion of rocky exoplanet remnants. One can also use the MS star chromospheric activity indices to correlate the star's activity with its age.

\subsection{Method}

Currently, there is only a small number of well studied WD + MS star in wide systems $([4,5])$ and with this in mind, we have created a sample of new common proper motion WD+MS star pairs. The WDs were selected from the SuperCOSMOS Science Archive following the technique described in [6]. This uses colour cuts and a reduced proper motion diagram to define a population of WDs. MS stars are from the Tycho catalog and are all brigth $(V<12)$, so that high resolution spectroscopy can be obtained for each object.

\subsection{Results}

We have observed 6 of our MS star components with the FEROS telescope, at the VLT. After reducing the spectra, we used the procedure described in [7] to determine effective temperatures, rotation and microturbulent velocities and chemical abundances for the targets.

As for the WD candidates, 50 of our initial sample of 160 were observed with IMACS, at the Magellan telescope and with X-shooter, at the VLT. Having done the spectral classification of our objects, we found that 10 were subdwarfs (main source of contamination) and the remaining are genuine WDs. Out of these latter, 5 show metal lines in their spectra (DAZ or DZ WDs). 


\section{Hot Planets and Cool Stars}

Ongoing work with the sample will include age estimation for the WDs. For the DAs that we have in the sample, this can be done by fitting models to their broad Balmer lines. Then, with the aid of model atmospheres, we estimate their effective temperatures and surface gravities. These atmoshperic parameters can ultimately be used to calculate the WDs cooling times and masses, providing us with an estimate of the system's age.

\section{References}

[1] D.J. Pinfield, H.R.A. Jones, P.W. Lucas, T.R. Kendall, S.L. Folkes, A.C. Day-Jones, R.J. Chappelle, I.A. Steele, MNRAS, 368, 1281 (2006), arXiv : astro-ph/0603320

[2] K.L. Cruz, I.N. Reid, J. Liebert, J.D. Kirkpatrick, P.J. Lowrance, AJ, 126, 2421 (2003), arXiv:astro-ph/0307429

[3] J.D. Kirkpatrick, I.N. Reid, J. Liebert, R.M. Cutri, B. Nelson, C.A. Beichman, C.C. Dahn, D.G. Monet, J.E. Gizis, M.F. Skrutskie, ApJ, 519, 802 (1999)

[4] S. Catalán, J. Isern, E. García-Berro, I. Ribas, C. Allende Prieto, A.Z. Bonanos, A\&A, 477, 213 (2008), 0710.1542

[5] J.K. Zhao, T.D. Oswalt, M. Rudkin, G. Zhao, Y.Q. Chen, AJ, 141, 107 (2011), 1101. 3257

[6] A.C. Day-Jones, D.J. Pinfield, R. Napiwotzki, B. Burningham, J.S. Jenkins, H.R.A. Jones, S.L. Folkes, D.J. Weights, J.R.A. Clarke, MNRAS, 388, 838 (2008), 0806.0015

[7] Y.V. Pavlenko, J.S. Jenkins, H.R.A. Jones, O. Ivanyuk, D.J. Pinfield, MNRAS, 422, 542 (2012), 1201.5099 